\title{
Central Nervous System Extraskeletal Osteosarcoma
}

National Cancer Institute

\section{Source}

National Cancer Institute. Central Nervous System Extraskeletal Osteosarcoma. NCI

Thesaurus. Code C7002.

An osteosarcoma arising from the brain or spinal cord. 